\title{
Microswimmers May Shepherd Large Liquid Volumes
}

\author{
According to simulations, a collection of tiny swimming particles can \\ corral a volume of liquid much larger than the sum of the volumes swept \\ along by each individual.
}

By Philip Ball

$\Lambda$ n object moving in water pulls some of the surrounding liquid along with it. Based on observations of such entrainment by individual microscopic particles, a team of researchers has now shown theoretically that the volume of fluid entrained by a group of such "swimmers" moving in concert can be much larger than the sum of the entrained volumes for the same number of isolated particles [1]. They suggest that this shepherding effect of a swarm of particles

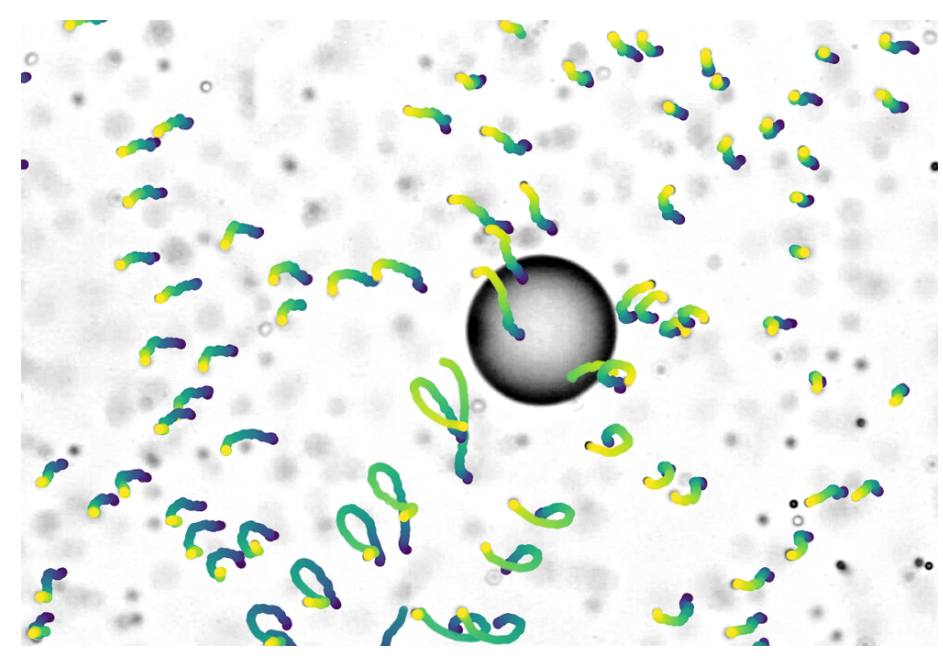

Pushing through a crowd. Trajectories of tracer particles entrained by a self-propelled particle (large sphere) are shown in this microscope image, colored to indicate timing, with dark blue corresponding to the starting time and yellow corresponding to the moment of the image. The sphere is moving upward, toward a point between the top center and upper-right corner (see videos below).

Credit: A. J. T. M. Mathijssen/Univ. of Pennsylvania could be used to transport drugs through the bloodstream or to control flows in microfluidic technology. This technique would allow the transport of more cargo than can be carried by packaging it in or on the swimmers.

Fluid entrainment is familiar from the way a car driving over a leaf-strewn road will pull some of the leaves with it in its wake. Entrainment is even stronger at smaller scales, where the fluid viscosity is more relevant and where a moving object may also push some of the fluid ahead of it.

Such effects can be significant for "active particles"-small objects that move under their own propulsion. Bacteria are the paradigmatic example of such microswimmers, some being propelled by spinning, corkscrew-like appendages. Several artificial microswimmers have now also been made, for example, asymmetric microparticles propelled by a gas-producing chemical reaction on one side of the particle [2].

Arnold Mathijssen of the University of Pennsylvania in Philadelphia and his co-workers have used experiments and calculations to look at how fluid entrainment by microswimmers can be altered by their collective motion. They first measured entrainment by individual microswimmers, watching them move in water containing smaller particles that acted as tracers of the fluid flow.

The microswimmers were micelles: blobs of oil about 48 micrometers across surrounded by soap-like surfactant molecules. As others have shown, these micelles will keep moving because of the asymmetric way that the surfactant molecules arrange themselves on the oily surface. 


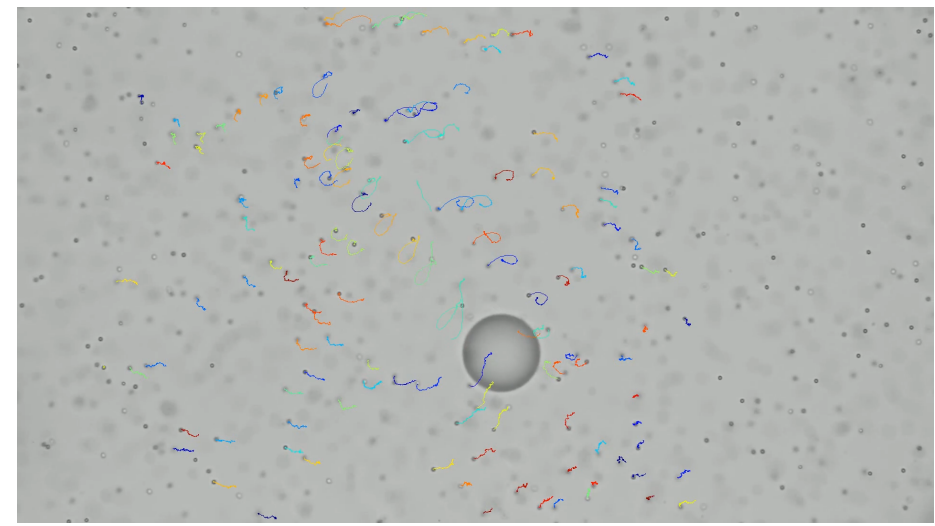

Tracer particles in water move as a self-propelled, 48-micrometer-wide droplet goes by.

Credit: C. Jin et al. [1]

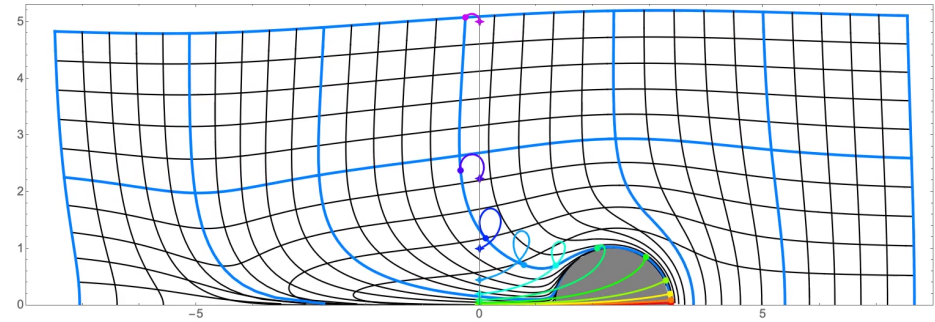

Simulation of particle entrainment by a self-propelled droplet. The deformations of the grid show the pattern of fluid flow around the particle, while the small colored particles are tracers. Some of these are initially pushed ahead of the moving droplet.

Credit: C. Jin et al. [1]

The researchers watched the particles moving individually through a suspension of hundreds of polymer tracer particles 2 micrometers in diameter. The fluid was confined between a pair of transparent plates whose separation was about the same as the particle width. They found that the observed tracer flow pattern around a microswimmer agreed well with theoretical predictions. The team then calculated what happens when many such active particles swim in a group.

The calculations showed that the volume of fluid the particles entrain can be about ten times greater en masse than the sum of the isolated particles' individual entrained volumes. That's partly because some of the fluid temporarily entrained and then

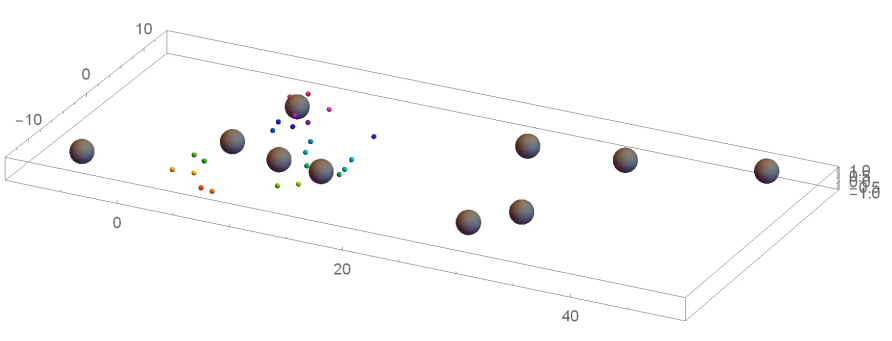

A school of self-propelled particles, confined to a narrow, slit-like channel, gradually pulls along a large volume of fluid (revealed here by colored tracer particles). Note that the entrained volume moves more slowly than the active particles.

Credit: C. Jin et al. [1]

shed by one particle can be "picked up" by those that come after. "The more swimmers, the better, we think," says Mathijssen. But he cautions that the team's model can't incorporate all of the interactions likely to occur between particles for very dense groups. What's more, the effects might be different for swimmers that move in different ways, for example, by wriggling rather than by advancing smoothly forward.

The team can't yet test these calculations for a group of swimmers. "Experiments with multiple swimmers would be very difficult because it is hard to track the particles [in a microscope] for a long enough time," says Mathijssen. "This may be possible in the future with more advanced 3D tracking methods."

All the same, the results predict that microswimmers could effectively herd a large amount of material dissolved or suspended in a fluid in a microchannel, for example, improving or directing the transport of drugs in blood vessels. "We think this effect will be useful for enhancing cargo transport by microrobots," says Mathijssen. The amount of cargo transported could be much greater than if it were simply loaded onto the individual microrobots, he says.

Soft-matter physicist Olivier Dauchot of ESPCI Paris calls the work "a truly nice and convincing study." Holger Stark, a specialist in biological physics at the Technical University of Berlin, adds that "the strength of the study is that they show this effect in a combined experimental and theoretical work." 
He agrees that it might be useful for transporting drugs or perhaps for removing pollution from fluids by using microrobotic swarms.

Condensed-matter physicist Julia Yeomans of the University of Oxford in the UK agrees that the results show promise but warns that, in practice, "it might be tricky to get the swimmers to all move in the same direction through any realistic setting, such as blood vessels."

Mathijssen suspects that such an effect might be exploited in living systems, for example, by bacteria whose swimming can spread fungal spores. This dispersion benefits the fungi that help the bacteria in other ways [3]. "Collective entrainment could be the driver for a number of symbiotic interactions between different species," he says.

Philip Ball is a freelance science writer in London. His latest book is The Modern Myths (University of Chicago Press, 2021).

\section{REFERENCES}

1. C. Jin et al., "Collective entrainment and confinement amplify transport by schooling microswimmers," Phys. Rev. Lett. 127, 088006 (2021).

2. W. F. Paxton et al., "Catalytic nanomotors: Autonomous movement of striped nanorods," J. Am. Chem. Soc. 126 (2004).

3. C. J. Ingham et al., "Mutually facilitated dispersal between the nonmotile fungus Aspergillus fumigatus and the swarming bacterium Paenibacillus vortex," Proc. Natl. Acad. Sci. U.S.A. 108, 19731 (2011). 